\title{
Simultaneous and Rapid Detection of Multiple Epimers and Isomers of Aspartyl Residues in Lens Proteins Using an LC-MS-MRM Method
}

\author{
Noriko Fujii,* Takumi Takata, Ingu Kim, and Toshiya Matsubara
}

Cite This: ACS Omega 2020, 5, 27626-27632

Read Online

ABSTRACT: Traditionally, studies of post translational modifications (PTMs) by mass analysis have been limited to modifications such as deamidation and oxidation that have a mass shift. Although Asp isomerization is an important PTM, the selective detection of Asp isomers by mass spectrometry was originally thought to be impossible due to the identical mass of the isomers. The recent development of an LC-MS-based method has facilitated rapid and accurate quantitative analysis of Asp isomers in long-lived proteins; however, because the quantification is based on the extracted ion chromatogram acquired by an MS1 scan, this methodology is not always efficient for detecting extremely low-abundance peptides in complex biological samples. In this paper, we evaluated Asp isomer-containing peptides of $\alpha \mathrm{A}$-crystallin present in tryptic digests of human lens samples with different degrees of protein aggregation and

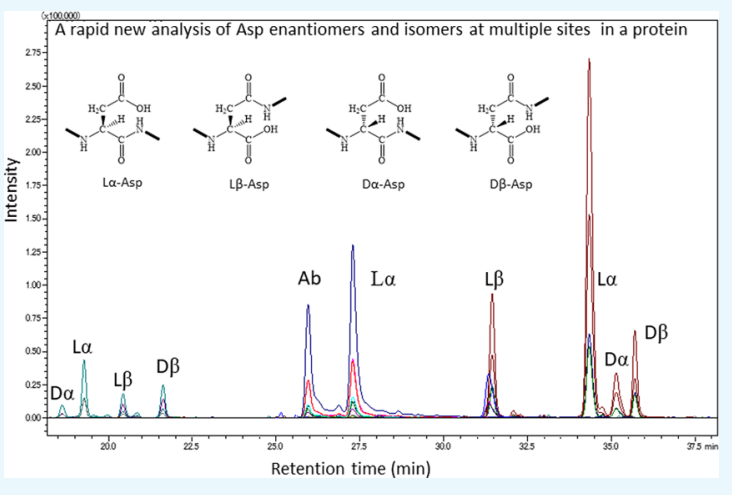
different ages using LC coupled with multiple reaction monitoring (MRM). In a single analysis, the LC-MRM method enabled three tryptic peptides containing isomers of Asp58, Asp91/92, and Asp151 to be detected simultaneously. The extent of isomerization and epimerization of these specific Asp sites in $\alpha \mathrm{A}$-crystallin increased with the progress of $\alpha$-crystallin aggregation. For the analysis of samples known to isomerize at specific Asp residues, MRM gives a more rapid, less laborious, and high-quality separation of Asp isomer-containing peptides relative to the previous MS1-based quantitative method.

\section{INTRODUCTION}

Post translational modifications (PTMs), such as oxidation, phosphorylation, deamidation, truncation, and disulfide bond formation, are known to increase during aging, inducing protein aggregation and insolubilization that may lead to agerelated disorders such as cataract and Alzheimer's disease. Studies of PTMs have increased alongside the progress in mass analysis technologies. For example, the +1 mass shift due to deamidation from asparagine (Asn) to aspartate (Asp) or from glutamine $(\mathrm{Gln})$ to glutamate $(\mathrm{Glu})$, and the +16 or +32 mass shift due to oxidation of methionine and tryptophan allow the detection of deamidation and oxidation sites in long-lived proteins in the lens and brain by mass analysis. Using liquid chromatography coupled with mass spectrometry (LC-MS), Wilmarth et al. identified 491 modification sites, including deamidation and S-methylation in aged lens proteins. ${ }^{1}$ By contrast, D-amino acids, which are generated in long-lived human proteins spontaneously through natural epimerization and isomerization, do not show a mass shift.

D-Aspartate was found initially identified in eye lenses, ${ }^{2-5}$ retina, ${ }^{6}$ teeth, ${ }^{7-9}$ arterial walls, ${ }^{10}$ artery, ${ }^{11}$ ligaments, ${ }^{12}$ bone, ${ }^{13,14}$ intervertebral disc, ${ }^{15}$ brain, ${ }^{16-19}$ and skin ${ }^{20-22}$ by the so-called diastereomer method, involving protein purification and digestion, followed by peptide sorting, sequencing, hydrolysis to amino acids and their derivatization, and lastly analysis of the diastereomers using high-performance liquid chromatography. ${ }^{23}$ Using this convoluted method, we found that Asp58 and Asp151 in $\alpha$ A-crystallin, ${ }^{4}$ Asp36 and Asp62 in $\alpha \mathrm{B}$-crystallin, ${ }^{3}$ and Asp 4 in $\beta \mathrm{B} 2$-crystallin ${ }^{5}$ from cataracts of elderly donors are highly inverted to D-isomers, while Hooi and Truscott additionally detected D-serine and D-threonine in cataract lenses. $^{24}$ The diastereomer method is accurate but time consuming for the analysis of D-amino acids in proteins. Furthermore, because epimerization of L-Asp to D-Asp is accompanied by simultaneous isomerization of the $\alpha$-linkage of the peptide bond to the $\beta$-linkage through the five-membered succinimide (Figure $\mathrm{S} 1$ ), the $\beta$-isomers must be analyzed by Edman degradation. Because these processes have several steps and require at least $1 \mathrm{mg}$ of the sample, many researchers have tried to use LC-MS to analyze D-amino acids including Asp isomers in proteins. Although LC-MS is a powerful tool, the analysis of $\mathrm{D}$-amino acid-containing peptides is not straightforward because the peptides have the same mass. However, recent advances and efforts including an O18 labeling

Received: August 30, 2020

Accepted: October 2, 2020

Published: October 16, 2020 

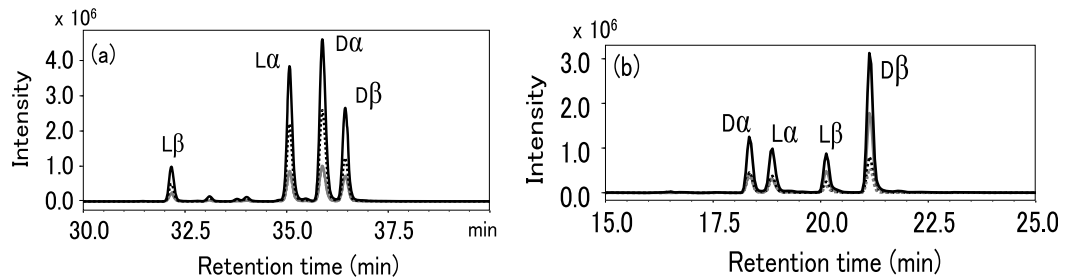

Figure 1. LC-MS-MRM chromatograms of the synthetic T6 peptide containing four Asp58 isomers (a) and the T18 peptide containing four Asp151 isomers (b). (a) LC-MS-MRM chromatogram of synthetic T6-L $\beta$, T6-L $\alpha$, T6-D $\alpha$, and T6-D $\beta$ peptide isomers, showing the elution order. Black line, TIC; black dots, y7 ion; gray line, y6 ion; gray dots, y4 ion (note that the gray solid and dotted lines overlap). (b) LC-MS-MRM chromatogram of synthetic T18-D $\alpha, \mathrm{T} 18-\mathrm{L} \alpha, \mathrm{T} 18-\mathrm{L} \beta$, and T18-D $\beta$ peptide isomers, showing the elution order. Black line, TIC; black dots, $\mathrm{y} 3$ ion; gray line, $\mathrm{y} 7$ ion; gray dots, $\mathrm{y} 4$ ion. The elution order of the T6 and T18 peptides isomers was determined by injecting individual standards; the corresponding chromatograms are shown in Figures S2 and S3, respectively.

strategy, $^{25}$ fragmentation techniques, ${ }^{26-28}$ and ion-mobility spectrometry ${ }^{29-31}$ have enabled the detection of D-amino acids in proteins by LC-MS. An excellent review by Jansson ${ }^{32}$ has recently covered the history of D-amino acid research and its analysis.

We have developed a method to more easily identify specific sites containing different Asp isomers ( $\alpha_{-}, \mathrm{L} \beta$-, $\mathrm{D} \alpha$-, and $\mathrm{D} \beta$-) in $\alpha$ A-crystallin by an MS1-based quantitative method using LC-MS/MS. ${ }^{33}$ The result clearly showed that Asp58, Asp91/ 92, and Asp151 residues are more prone to isomerization than Asp76 or Asp84. In the approach, tryptic peptides of the protein of interest are separated by LC and identified by MS/ MS analysis, and multiple peaks with the same amino acid sequence are extracted as potential peptides containing Asp isomers. The different Asp isomers in peptides are then identified by matching to the retention time of reference peptides containing the four different Asp isomers ${ }^{33}$ or using isomer-specific commercial enzymes. ${ }^{34}$ Recently, the analysis of Asp isomerization and epimerization has been further developed for lens protein ${ }^{35}$ and also expanded to myelin basic protein from multiple sclerosis ${ }^{36}$ using LC-MS/MS. The MS1based quantitative method employed in the above reports can successfully detect hot spots of Asp isomerization and epimerization in tissues in a short time relative to previous studies; however, its application to minor components in a sample is less precise. Furthermore, it is not efficient for the comparison of multiple similar samples collected under different conditions (e.g., age, sex, and tissue parts) but with the same hot spots of isomerization and epimerization.

The long-lived lens protein $\alpha$-crystallin is a hetero-oligomer of 40-50 subunits of $\alpha \mathrm{A}$ and $\alpha \mathrm{B}$ (each $\sim 20 \mathrm{kDa}$ ) and interacts with $\beta$ - and $\gamma$-crystallins to maintain transparency. Although $\alpha$ crystallin is a soluble protein, it forms aggregates of high molecular weight (HMW) and eventually becomes water insoluble (WI) during aging. These changes are considered to underlie the development of cataract. Because their turnover in the lens is very low, the ability of crystallins to maintain their native conformation is crucial. $\alpha$-Crystallin undergoes various PTMs over time, including isomerization and epimerization, and these modifications are considered to cause the aggregation of $\alpha$-crystallin.

In this study, we describe the rapid and high-sensitivity analysis of multiple sites of Asp isomers in human lens $\alpha \mathrm{A}$ crystallin based on a targeted proteomics approach by LC-MS/ MS coupled with multiple reaction monitoring (LC-MSMRM). Applying this method to soluble $\alpha$-crystallin (SAC), HMW and WI fractions derived from lenses of donors of different ages, we report for the first time that isomerization and epimerization of three specific sites of Asp residues in $\alpha \mathrm{A}$ crystallin increase as the size of $\alpha$-crystallin aggregate increases.

\section{RESULTS AND DISCUSSION}

2.1. Identification of Asp58 Isomers in Synthetic T6 Peptides by LC-MS/MS-MRM. Figure 1a shows the LC-MSMRM chromatogram of a solution of synthetic T6 peptides containing the four Asp isomers: T6-L $\beta, \operatorname{TVLD}^{58}(\mathrm{~L} \beta)$ SGISEVR); T6-L $\alpha, \quad\left(\mathrm{TVLD}^{58}(\mathrm{~L} \alpha)\right.$ SGISEVR); T6-D $\alpha$, $\left(\mathrm{TVLD}^{58}(\mathrm{D} \alpha)\right.$ SGISEVR); and $\mathrm{T} 6-\mathrm{D} \beta, \quad\left(\mathrm{TVLD}^{58}(\mathrm{D} \beta)-\right.$ SGISEVR). The precursor ions of the peptides were obtained as divalent ions (TVLD ${ }^{58}$ SGISEVR; $[\mathrm{M}+2 \mathrm{H}]^{2+}=588.3$ ). Four T6 isomeric peptides were individually applied to LCMS-MRM in advance (Figure S2), which established the elution order of the T6-L $\beta$, T6-L $\alpha$, T6-D $\alpha$, and T6-D $\beta$ peptides in the mixture. For each of the four T6 peptides, the peaks corresponding to the total ion chromatogram (TIC) (black line), $y_{7}$ (black dots), $y_{6}$ (gray line), and $y_{4}$ (gray dots) ions clearly overlapped, showing that the peptide isomers were identified accurately. All identified fragment ions were detected as monovalent ions $\left(y_{7}, 747.4 ; y 6,660.37 ; y_{4}, 490.26\right)$, as shown in Table 1. Baseline separation of the T6 isomers was successfully accomplished for an injection of $1 \mathrm{fmol}$ of the sample.

2.2. Identification of Asp151 Isomers in Synthetic T18 Peptides by LC-MS/MS-MRM. Figure 1 b shows the LC-MS-

Table 1. Three Peptide Isomers of Human $\alpha$ A-Crystallin and Their MRM Transition

\begin{tabular}{|c|c|c|}
\hline $\begin{array}{l}\text { peptide } \\
\text { name }\end{array}$ & peptide sequence & MRM transitions \\
\hline T6-L $\alpha$ & TVL-L $\alpha \mathrm{D}^{58} \mathrm{SGISEVR}$ & y7: $588.32>747.40$ \\
\hline T6-L $\beta$ & TVL-L $\beta \mathrm{D}^{58}$ SGISEVR & y6: $588.32>660.37$ \\
\hline T6-D $\alpha$ & TVL-D $\alpha \mathrm{D}^{58}$ SGISEVR & $y 4: 588.32>490.26$ \\
\hline T6-D $\beta$ & $\begin{array}{l}\text { TVL-D } \beta \mathrm{D}^{58} \text { SGISEVR }[\mathrm{M}+2 \mathrm{H}]^{2+}: \\
588.32\end{array}$ & \\
\hline $\mathrm{T} 18-\mathrm{L} \alpha$ & IQTGL-L $\alpha \mathrm{D}^{151}$ ATHAER & y7: $437.89>400.20^{b}$ \\
\hline $\mathrm{T} 18-\mathrm{L} \beta$ & IQTGL-L $\beta \mathrm{D}^{151} \mathrm{ATHAER}$ & $y 4: 437.89>512.26$ \\
\hline $\mathrm{T} 18-\mathrm{D} \alpha$ & IQTGL-D $\alpha \mathrm{D}^{151}$ ATHAER & $y 3: 437.89>375.2$ \\
\hline $\mathrm{T} 18-\mathrm{D} \beta$ & $\begin{array}{l}\text { IQTGL-D } \beta \mathrm{D}^{151} \text { ATHAER } \\
{[\mathrm{M}+3 \mathrm{H}]^{3+}: 437.89}\end{array}$ & \\
\hline $\mathrm{T} 11-\mathrm{L} \alpha$ & VQ-L $\alpha \mathrm{D}^{91}-\mathrm{L} \alpha \mathrm{D}^{92} \mathrm{FVEIHGK}$ & $y 7: 429.55>415.23^{b}$ \\
\hline $\mathrm{T}_{\text {Overall }}$ & $\begin{array}{l}\text { VQ- }(\mathrm{L} / \mathrm{D}) \alpha \beta \text { D } 91-(\mathrm{L} / \mathrm{D}) \\
\alpha \beta \mathrm{D}^{92} \mathrm{FVEIHGK}[\mathrm{M}+3 \mathrm{H}]^{3+}: \\
429.55\end{array}$ & $\begin{array}{l}\text { y6: } 429.55>682.39 \\
\text { y5: } 429.55>583.32 \\
\text { y4: } 429.55>454.28 \\
\text { y3: } 429.55>341.19\end{array}$ \\
\hline
\end{tabular}

a Overall 15 specific isomers were possible; the actual isomer(s) that were present were not determined. ${ }^{b}$ Divalent ion. 
MRM chromatogram of a solution of synthetic T18 peptides containing four Asp151 isomers: T18-D $\alpha$, (IQTGLD ${ }^{151}(\mathrm{D} \alpha)$ ATHAER); T18-L $\alpha$, (IQTGLD ${ }^{151}(\mathrm{~L} \alpha)$ ATHAER); T18-L $\beta$, (IQTGLD $^{151}$ (L $\left.\beta\right)$ ATHAER); and T18-D $\beta$ IQTGLD $^{151}$ (D $\left.\beta\right)$ ATHAER). The precursor ions of the peptides were obtained as trivalent ions (IQTGLD ${ }^{151}$ ATHAER; $[\mathrm{M}+3 \mathrm{H}]^{3+}=$ 437.89). Four T18 isomeric peptides were individually applied to LC-MS-MRM in advance (Figure S3), which established the elution order of the mixture of the T18 four isomeric peptides as $\mathrm{T} 18-\mathrm{D} \alpha, \mathrm{T} 18-\mathrm{L} \alpha, \mathrm{T} 18-\mathrm{L} \beta$, and $\mathrm{T} 18-\mathrm{D} \beta$. The peaks corresponding to the TIC (black line), $y_{4}$ (gray dots), $y_{3}$ (black dots), and $\mathrm{y}_{7}$ (gray line) ions of the four T18 peptide isomers clearly overlapped, showing that the peptide isomers were identified accurately. All identified fragment ions were detected as monovalent ions except for the $y_{7}$ divalent ion $\left(y_{7}\right.$, $\left.400.2\left([\mathrm{M}+2 \mathrm{H}]^{2+}\right) ; \mathrm{y} 4,512.5 ; \mathrm{y}_{3}, 375.2\right)$, as shown in Table 1. Baseline separation of the T18 isomers was successfully accomplished for an injection of $1 \mathrm{fmol}$ of the sample.

2.3. New Survey of Isomeric Asp Residues in SAC, HMW, and WI Fractions of Human $\alpha A-C r y s t a l l i n$ Using LC-MS/MS-MRM. Figure $2 a-c$ shows a typical LC-MS-MRM chromatogram of respective $\mathrm{T} 6, \mathrm{~T} 11$, and $\mathrm{T} 18$ isomeric peptides obtained from trypsin digestion of $\alpha \mathrm{A}$-crystallin in the SAC fraction derived from the lens of a 20 -year-old donor. Although MRM transitions were valid for the tryptic T1, T6, T9, T10, T11, T13, and T18 peptides of $\alpha$ A-crystallin (Table

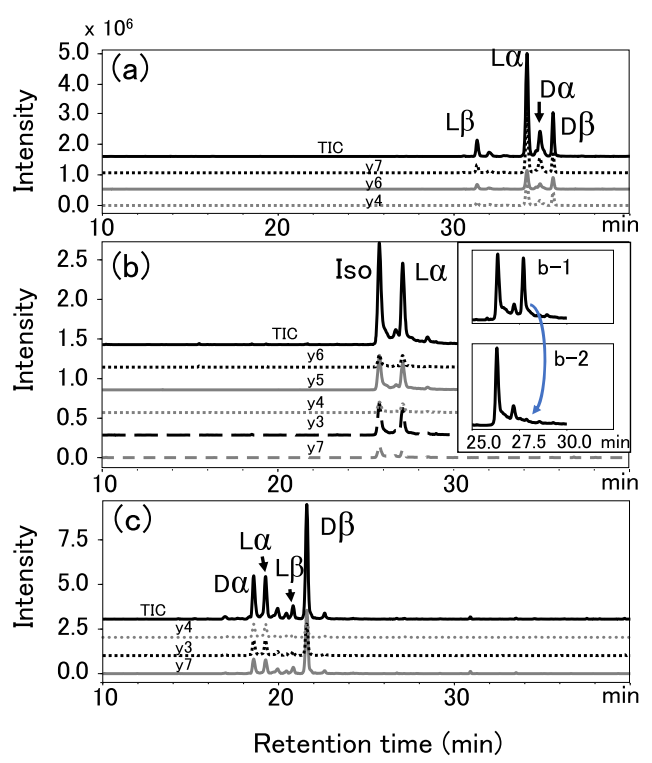

Figure 2. Typical LC-MS-MRM chromatogram of (a) T6 (Asp58), (b) T11 (Asp91/92), and (c) T18 (Asp151) peptide isomers of $\alpha$ Acrystallin in the SAC fraction derived from the lens of a 20 -year-old donor. (a) LC-MS-MRM chromatogram of T6-L $\beta$, T6- $\mathrm{L} \alpha$, T6-D $\alpha$, and T6-D $\beta$ peptide isomers. Black line, TIC; black dots, $\mathrm{y} 7$ ion; gray line, y6 ion; gray dots, y4 ion. (b) LC-MS-MRM chromatogram of the T11-L $\alpha$ peptide and T11-isomer (T-11-Iso) peptides. Black line, TIC; black dots, y6 ion; gray line, y5 ion; gray dots, y4 ion; black broken line, y3 ion; gray broken line, y7 ion. Identification of the T11-L $\alpha$ peptides was determined by analysis before (b-1) and after (b-2) Asp$\mathrm{N}$ treatment, as described in Section 2.3. (c) LC-MS-MRM chromatogram of $\mathrm{T} 18-\mathrm{D} \alpha, \mathrm{T} 18-\mathrm{L} \alpha, \mathrm{T} 18-\mathrm{L} \beta$, and $\mathrm{T} 18-\mathrm{D} \beta$ peptide isomers. Black line, TIC; gray dots, y4 ion; black dots, $\mathrm{y} 3$ ion; gray line, $\mathrm{y} 7$ ion. Identification of the T6 and T18 isomeric peptides was determined by matching the LC retention times of their synthetic peptides, as shown in Figure 1a,b.
S1), the actual peptides including Asp isomers, in other words, those showing multiple peaks with high MRM transitions, were limited to the T6, T11, and T18 peptides, whereas the MRM transitions of $\mathrm{T} 1, \mathrm{~T} 9, \mathrm{~T} 10$, and $\mathrm{T} 13$ were too low to detect. In a single analysis, MRM transitions enabled us to monitor the T6, T11, and T18 peptides containing Asp isomers simultaneously (Table 1 ).

Identification of the T6 (Figure 2a) and T18 (Figure 2c) isomeric peptides was determined by matching the LC retention times of their synthetic peptides, as shown in Figure la,b. However, we found that Asp residues of T11 were also highly inverted to Asp isomers, consistent with the previous study of Lyon et al. ${ }^{35}$ As shown in Figure $2 b$, the T11 peptide was separated by LC-MS-MRM into two major peaks and one minor peak. In this case, however, it was difficult to identify the normal and isomeric T11 and peptides by comparison to a synthetic peptide because 16 reference peptides (VQ-(L) D) $\left.\alpha \beta \mathrm{D}^{91}-(\mathrm{L} / \mathrm{D}) \alpha \beta \mathrm{D}^{92} \mathrm{FVEIHGK}\right)$ corresponding to each of the four isomers at the two Asp residues would be needed. Therefore, we identified normal peptides versus other isomeric peptides by a previous method, ${ }^{34}$ in which an Asp-N enzyme is used to hydrolyze the peptide bond on the $\mathrm{N}$-terminus of only an $\mathrm{L} \alpha$-Asp residue, and thus differentiates between peptides containing $\mathrm{L} \alpha$-Asp and those containing other isomers of Asp. The LC-MS-MRM spectrum of the T11 peptide before (Figure 2b-1) and after (Figure 2b-2) Asp-N treatment is shown in the inset of Figure $2 \mathrm{~b}$. The second major peak disappeared after Asp-N treatment, showing that it was the normal $\mathrm{L} \alpha$-Asp-containing T11 peptide. The intensities of the first major peak and the minor peak did not change, indicating that these peaks correspond to peptides containing Asp isomers at 91 and/or 92 . The peak areas showed that the ratio of the T11-iso peptides to T11-L $\alpha$ peptide was 1.0. The respective fragment (y) ions of the T6, T11, and T18 peptides clearly overlapped, showing that identification of the peptides was achieved easily, and the ratio of the Asp isomers could be calculated accurately by the baseline separation of the chromatogram.

Figure 3 shows typical LC-MS-MRM chromatograms (TIC) of the T6, T11, and T18 peptide isomers of $\alpha \mathrm{A}$-crystallin in the SAC, HMW, and WI fractions of lens from a 20-year-old donor. The degree of the aggregation increased in SAC, HMW, and WI fractions. For T6, the T6-L $\alpha$-Asp peptide had the largest peak in all fractions; however, the amount of the T6-L $\beta$ and T6-D $\beta$ peptides considerably increased from the SAC to the HMW fraction and was highest in the WI fraction (Figure 3). In all fractions, the T6-D $\beta$ peptide was the most abundant among the abnormal peptide isomers. For the T11 (Asp91/92containing) peptide, T11-iso peptides were detected in all fractions, and the amount of T11-iso peptides increased with the increasing aggregation of $\alpha \mathrm{A}$-crystallin in the fractions, that is, $\mathrm{SAC}<\mathrm{HMW}=\mathrm{WI}$ (Figure 3). For the T18 (Asp151containing) peptide of $\alpha \mathrm{A}$-crystallin, the most abundant isomer was not the normal T18-L $\alpha$ peptide but the abnormal T18-D $\beta$ peptide in all fractions (Figure 3). Thus, Asp151 is more prone to isomerization to the $\mathrm{D} \beta$-Asp isomer as compared with Asp58.

Figure 4 shows the ratios of the four isomers present at Asp58, Asp151, and Asp91/92 of $\alpha$ A-crystallin in the SAC, HMW, and WI fractions of lenses from 20-, 56-, and 76-yearold donors. For all samples, the relative amounts of the three abnormal isomers at Asp58, Asp91/92, and Asp151 were calculated from the LC-MS-MRM (TIC) peak areas by setting 
(a) T6 peptide

(b) T11 peptide

(c) T18 peptide
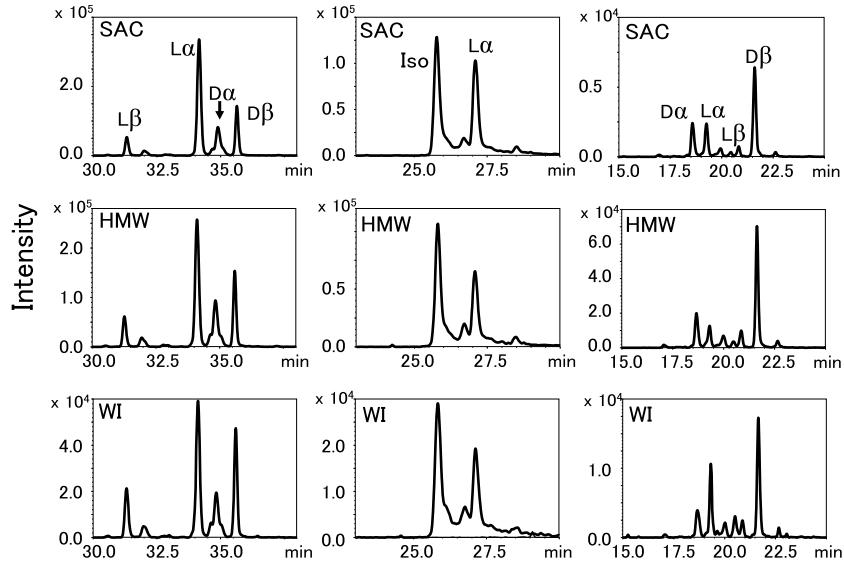

Retention time ( $\mathrm{min})$

Figure 3. LC-MS-MRM chromatograms (TIC) of T6 (a), T11 (b), and $\mathrm{T} 18(\mathrm{c})$ isomeric peptides of $\alpha \mathrm{A}$-crystallin due to isomerization of Asp58, Asp91/92, and Asp151, respectively, in the SAC, HMW, and WI fractions of lens derived from a 20 -year-old donor. For comparison purposes, the TICs of T6, T11, and T18 from the SAC fraction shown in $a-c$ have been replotted from Figure 2 . the peak area of the $L \alpha$-Asp-containing peptide as 1.0. The relative amounts of all four isomers were then used to calculate the percentage distribution of isomers in each sample. For Asp58 (Figure 4a), the amount of normal $\mathrm{L} \alpha$-Asp58 decreased with the progression of aggregation (i.e., $\mathrm{SAC}<\mathrm{HMW}<\mathrm{WI}$ ) for lens samples from all age groups, whereas the amount of abnormal $\mathrm{L} \beta$-, $\mathrm{D} \alpha$-, and $\mathrm{D} \beta$-Asp58 isomers increased in the order SAC $<\mathrm{HMW}<\mathrm{WI}$. Among the abnormal isomers, the $\mathrm{D} \beta$-Asp isomer was predominant in the lens sample of all ages. For Asp151 (Figure 4b), the most abundant isomer was the $\mathrm{D} \beta$-Asp isomer, and the ratio of $\mathrm{D} \beta$-Asp to $\mathrm{L} \alpha$-Asp was greater than 1.0 in all fractions of lens from all age groups. Thus, Asp151 seems to be more prone to isomerization and epimerization to the $\mathrm{D} \beta$-Asp isomer relative to Asp58, as noted above. For Asp91/92 (Figure 4c), the relative amount of abnormal Asp91/92 isomers in the fractions of lens from all ages increased in the order of the progression of aggregation: $\mathrm{SAC}<\mathrm{HMW}<\mathrm{WI}$ fractions. In particular, the ratio of abnormal isomers to normal L-Asp at Asp91/92 was greater than 1.0 in the fractions of lens from the 20 -year-old donor. A similar tendency was observed for the lens samples from the 56- and 76-year-old donors.

Taken together, our observations clearly show that the extent of isomerization and epimerization of Asp58 increases with increasing aggregation of $\alpha \mathrm{A}$-crystallin, and the isomer-
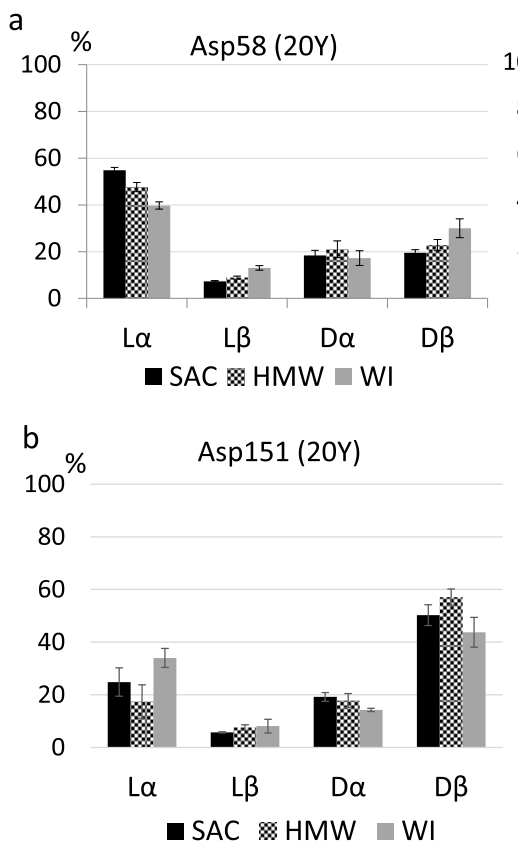

$\mathrm{C}$

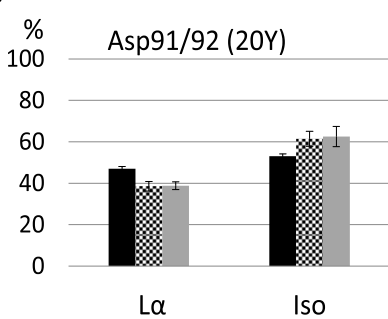

$\square \mathrm{SAC} \times \mathrm{HMW} \square \mathrm{WI}$

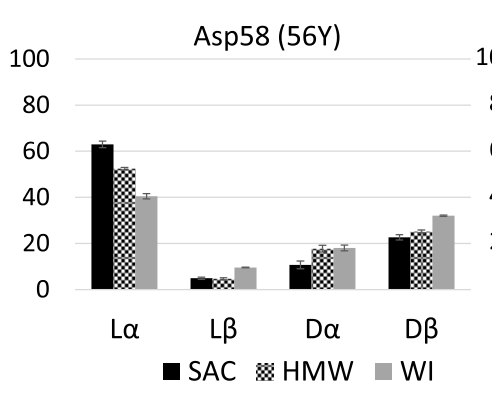

Asp151 (56Y)

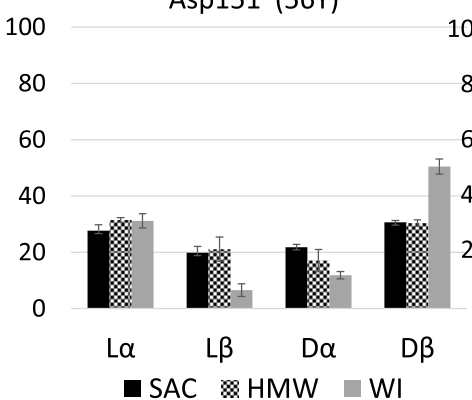

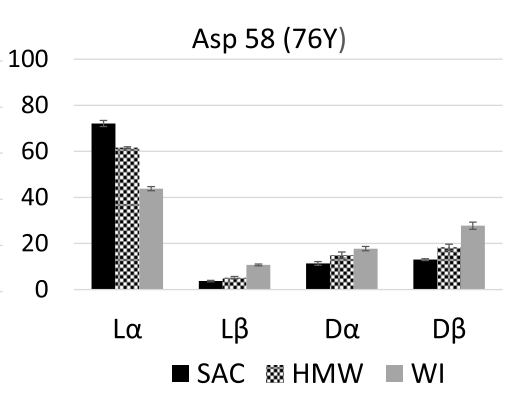

Asp151 (76Y)

00
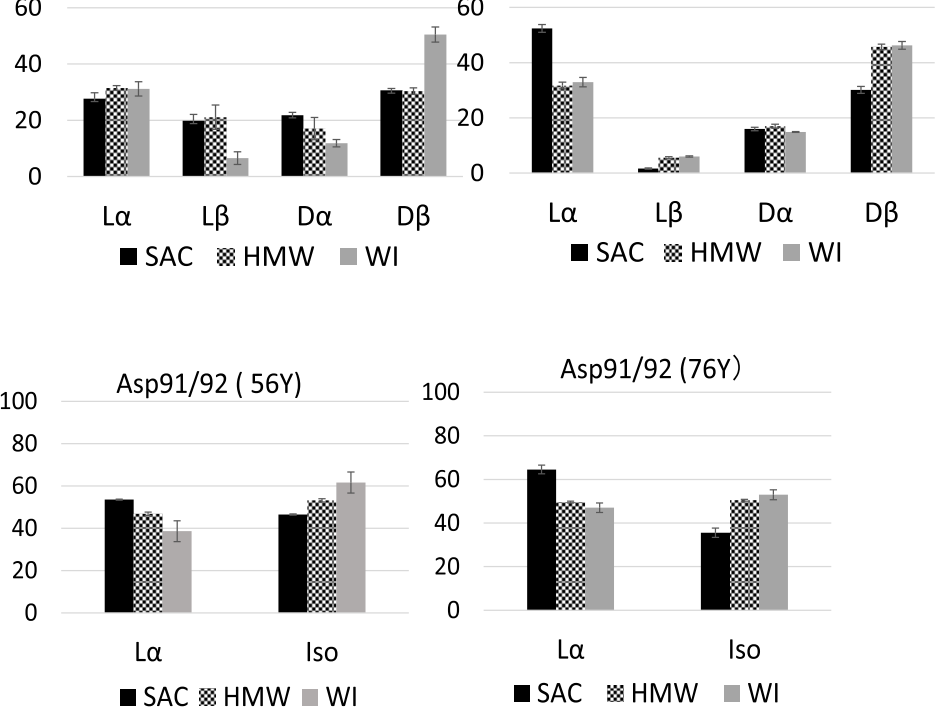

Figure 4. Relative amounts of Asp isomers in human $\alpha$ A-crystallin in SAC, HMW, and WI fractions of lens derived from 20-, 56-, and 76-year-old donors. (a) Asp58, (b) Asp151, and (c) Asp91/92. Error bars represent the standard deviation of triplicate LC-MS-MRM analyses. 
ization of Asp151 proceeds more rapidly than Asp58. The extent of the isomerization may not be related only to protein solubility in lens aged over 20 years because Asp151 is located in the structural flexible $\mathrm{C}$-terminal region of $\alpha \mathrm{A}$-crystallin. To elucidate the relationship between age and Asp isomerization, further examination of multiple samples of lens from several donors of different ages is needed.

\section{CONCLUSIONS}

The previous MS1-based method by LC-MS/MS is useful for the analysis of amino acid isomers and epimers in a protein, but it has problems such as the chromatographic separation and the length of time for analysis. The new detection and relative quantification scheme for peptide isomers described in the present study utilizes MRM-based targeted proteomics technology to improve chromatographic separation and facilitate identification by the automated detection of common fragment ions. Using this method, we were able to identify multiple sites of Asp isomerization in $\alpha \mathrm{A}$-crystallin for the first time. This targeted analysis by MRM will be a powerful tool to study the isomerization and epimerization of Asp in proteins of multiple samples.

\section{EXPERIMENTAL METHODS}

4.1. Enzymes. Sequencing-grade modified trypsin and endoprotease Asp-N were purchased from Promega (Madison) and Roche (Basel, Switzerland), respectively. Trypsin was dissolved in $50 \mathrm{mM}$ Tris/ $\mathrm{HCl}(\mathrm{pH} 7.5)$ buffer to a final concentration of $100 \mu \mathrm{g} / \mathrm{mL}$. Asp-N was dissolved in $10 \mathrm{mM}$ Tris/ $\mathrm{HCl}(\mathrm{pH} 7.5)$ to a final concentration of $40 \mu \mathrm{g} / \mathrm{mL}$. These enzymes were stored at $-80{ }^{\circ} \mathrm{C}$ before enzymatic digestion.

4.2. Preparation of SAC, HMW, and WI Proteins from Human Lenses. Human lenses, approved for research use, were provided by Dr. Kamei of Meijo University, Japan. Lens samples from individuals aged 20,56, and 76 years (one lens each) were homogenized in $20 \mathrm{mM}$ Tris/ $\mathrm{HCl}, \mathrm{pH} \mathrm{7.8,150}$ $\mathrm{mM} \mathrm{NaCl}$ buffer and fractionated into water-insoluble (WI) and water-soluble (WS) fractions by centrifugation at $16000 \mathrm{~g}$ for $20 \mathrm{~min}$ at $4{ }^{\circ} \mathrm{C}$. The WS fraction was applied to sizeexclusion chromatography (SEC) using a HiPrep 16/60 Sephacryl S-300 high-resolution column (GE Healthcare, Piscataway, NJ), equilibrated with the above buffer to separate high-molecular-weight (HMW) protein and soluble $\alpha$-crystallin (SAC) fractions. Samples were eluted at a flow rate of 0.5 $\mathrm{mL} / \mathrm{min}$ with monitoring at $280 \mathrm{~nm}$.

4.3. Preparation of Tryptic Peptides from SAC, HMW, and WI Proteins. The WI proteins were first dissolved in $8 \mathrm{M}$ urea, $50 \mathrm{mM}$ Tris $/ \mathrm{HCl}$, $\mathrm{pH} 7.8$, and $1 \mathrm{mM} \mathrm{CaCl}_{2}$; the urea concentration was then diluted to less than $1 \mathrm{M}$ in $50 \mathrm{mM}$ Tris/HCl, $\mathrm{pH} 7.8,1 \mathrm{mM} \mathrm{CaCl} \mathrm{C}_{2}$ buffer before enzymatic digestion. The WI, HMW, and SAC fractions were digested with trypsin for $17 \mathrm{~h}$ at $37^{\circ} \mathrm{C}$ in $50 \mathrm{mM}$ Tris/ $\mathrm{HCl}, \mathrm{pH} 7.8,1$ $\mathrm{mM} \mathrm{CaCl}{ }_{2}$ buffer at an enzyme-to-substrate ratio of 1:50 (w/ $w)$. Each enzymatic digestion was stopped by the addition of $0.1 \%$ formic acid and stored at $-80{ }^{\circ} \mathrm{C}$ until analysis.

4.4. Rapid Detection of Asp Isomers in Human $\alpha$ ACrystallin by a High-Sensitivity Targeted Proteomics Approach using Microflow LC-MS/MS. Solutions containing $0.1-1 \mu \mathrm{g}$ of peptides were quantified by MRM measurement with microflow electrospray ionization (ESI). LC separation was carried out on a microflow LC system (Nexera
Mikros X2 Shimadzu, Japan; including trap and elute system) using a combination of a C18 column (L-column2 ODS, 0.3 $\mathrm{mm} \times 150 \mathrm{~mm}, 2 \mu \mathrm{m}$ CERI, Japan) for analysis and a C8 column (L-column C8, $0.3 \mathrm{~mm} \times 5 \mathrm{~mm}, 5 \mu \mathrm{m}$ CERI, Japan) as a trap. The binary mobile phase compositions were $0.1 \%$ formic acid/water (mobile phase A) and $0.1 \%$ formic acid/ acetonitrile (mobile phase $\mathrm{B}$ ). The linear gradient was $0-15 \%$ phase B for $40 \mathrm{~min}$ at a flow rate of $10 \mu \mathrm{L} / \mathrm{min}$, and a column temperature of $40{ }^{\circ} \mathrm{C}$. All samples were filtered through a 0.45 $\mu \mathrm{m}$ filter (Millex, Millipore) before analysis. Asp-containing peptides were detected by the MRM method using a triple quadrupole mass spectrometer (LCMS-8060 with Micro ESI8060; Shimadzu, Tokyo, Japan). Transitions covered more than four fragment ions for identification of amino acid sequences. Prior to sample analysis, it was necessary to survey the valid MRM transitions for all potential tryptic peptides of $\alpha \mathrm{A}$-crystallin: trypsin digestion of $\alpha \mathrm{A}$-crystallin was expected to yield 20 peptides, T1-T20 (Figure S4). T1-T20 correspond to known tryptic peptides of $\alpha \mathrm{A}$-crystallin numbered from the $\mathrm{N}$-terminus starting with T1. The MRM method for each Aspcontaining peptide was created with Skyline software (University of Washington, Seattle, WA) and Lab Solutions software (Shimadzu, Japan). The MRM chromatograms for fragment ions of each peptide were acquired and used as total ion chromatograms in an ESI positive mode with the transitions shown in Table S1. Among all tryptic peptides, multiple peaks with high MRM transitions were limited to T6, T11, and T18; therefore, we analyzed only these three peptide isomers, as shown in Table 1.

4.5. Synthesis of T6 and T18 Peptides Containing Four Different Asp Isomers. Reference peptides of T6 and T18 containing the four different Asp isomers were synthesized by Fmoc solid-phase chemistry using an automated solid-phase peptide synthesizer (PSSM-8, Shimadzu, Japan) according to the previous method. ${ }^{33}$ The synthetic peptides were stored at $-80{ }^{\circ} \mathrm{C}$ until analysis. All of the peptides were stable and did not isomerize during the timescale of the experiments.

4.6. Identification of the Four Isomers of Asp58 and Asp151 in Respective T6 and T18 Reference Peptides by LC-MS-MRM. Synthetic T6 peptides containing the four different isomers of Asp58 were each applied to LC-MS-MRM, and the individual retention times were used to identify peaks in mixtures of the four Asp isomers applied to LC-MS-MRM. LC-MS conditions were the same as those described in Section 4.4. T18 peptides containing the four different isomers of Asp151 were synthesized and used for analysis in the same way.

4.7. Determination of Normal L $\alpha$-Asp Residues in the T11 Peptide (T11-L $\alpha: V Q-L \alpha D^{91}-\mathrm{L} \alpha \mathrm{D}^{92}$ FVEHGK) by the Asp-N Enzyme. Because T11 peptides contain two Asp residues, it would be time consuming to synthesize 16 different isomeric peptides. Asp-N hydrolyzes peptide bonds on the $\mathrm{N}$ terminal side of only $\mathrm{L} \alpha$-Asp residues in a peptide. Therefore, we differentiated between normal $\mathrm{L} \alpha$-Asp-containing peptide and peptide isomers using Asp-N, as described previously. ${ }^{34}$ In brief, tryptic peptides of the sample solution were digested with Asp-N at an enzyme/peptide ratio (w/w) of 1:20 for $17 \mathrm{~h}$ at $37{ }^{\circ} \mathrm{C}$. The peptides of before and after Asp-N treatment were analyzed by LC-MS-MRM. Only the T11-L $\alpha$ peptide is expected to disappear while T11-iso peptides remain on the chromatogram. LC-MS conditions were the same as those described in Section 4.4. 


\section{ASSOCIATED CONTENT}

\section{(s) Supporting Information}

The Supporting Information is available free of charge at https://pubs.acs.org/doi/10.1021/acsomega.0c04197.

Additional data including LC-MS-MRM chromatograms of synthetic T6 and T18 peptides and the amino acid sequences of human $\alpha \mathrm{A}$-crystallin and the MRM transition are provided (PDF)

\section{AUTHOR INFORMATION}

\section{Corresponding Author}

Noriko Fujii - Institute for Integrated Radiation and Nuclear Science, Kyoto University, Osaka 590-0494, Japan; (1) orcid.org/0000-0002-4009-342X; Phone: +81-72-4512496; Email: nfujii@rri.kyoto-u.ac.jp

\section{Authors}

Takumi Takata - Institute for Integrated Radiation and Nuclear Science, Kyoto University, Osaka 590-0494, Japan

Ingu Kim - Institute for Integrated Radiation and Nuclear Science, Kyoto University, Osaka 590-0494, Japan

Toshiya Matsubara - Shimadzu Corporation, Kyoto 604-8511, Japan

Complete contact information is available at:

https://pubs.acs.org/10.1021/acsomega.0c04197

\section{Author Contributions}

This paper was written through the contributions of all authors.

\section{Notes}

The authors declare no competing financial interest.

\section{ACKNOWLEDGMENTS}

This work was supported by a grant from the Ministry of Education, Culture, Sports, Science and Technology of Japan (25288075).

\section{REFERENCES}

(1) Wilmarth, P. A.; Tanner, S.; Dasari, S.; Nagalla, S. R.; Riviere, M. A.; Bafna, V.; Pevzner, P. A.; David, L. L. Age-related changes in human crystallins determined from comparative analysis of posttranslational modifications in young and aged lens: does deamidation contribute to crystallin insolubility? J. Proteome Res. 2006, 5, 25542566.

(2) Masters, P. M.; Bada, J. L.; Zigler, J. S., Jr. Aspartic acid racemisation in the human lens during ageing and in cataract formation. Nature 1977, 268, 71-73.

(3) Fujii, N.; Ishibashi, Y.; Satoh, K.; Fujino, M.; Harada, K. Simultaneous racemization and isomerization at specific aspartic acid residues in alpha B-crystallin from the aged human lens. Biochim. Biophys. Acta, Protein Struct. Mol. Enzymol. 1994, 1204, 157-163.

(4) Fujii, N.; Satoh, K.; Harada, K.; Ishibashi, Y. Simultaneous stereoinversion and isomerization at specific aspartic acid residues in alpha A-crystallin from human lens. J. Biochem. 1994, 116, 663-669.

(5) Fujii, N.; Kawaguchi, T.; Sasaki, H.; et al. Simultaneous stereoinversion and isomerization at the Asp-4 residue in betaB2crystallin from the aged human eye lenses. Biochemistry 2011, 50, $8628-8635$.

(6) Kaji, Y.; Oshika, T.; Takazawa, Y.; Fukayama, M.; Takata, T.; Fujii, N. Localization of D-beta-aspartic acid-containing proteins in human eyes. Invest. Ophthalmol. Visual Sci. 2007, 48, 3923-3927.

(7) Masters, P. M. Stereochemically altered noncollagenous protein from human dentin. Calcif. Tissue Int. 1983, 35, 43-47.
(8) Masuda, W.; Nouso, C.; Kitamura, C.; Terashita, M.; Noguchi, T. D-Aspartic acid in bovine dentine non-collagenous phosphoprotein. Arch. Oral Biol. 2002, 47, 757-762.

(9) Ohtani, S.; Yamamoto, T.; Sugimoto, H.; Sashima, M.; Satoh, M. Age-related changes in the D-aspartic acid content of the teeth of the senescence-accelerated mouse. Arch. Oral Biol. 2000, 45, 13-18.

(10) Powell, J. T.; Vine, N.; Crossman, M. On the accumulation of $\mathrm{D}$-aspartate in elastin and other proteins of the ageing aorta. Atherosclerosis 1992, 97, 201-208.

(11) Dobberstein, R. C.; Tung, S. M.; Ritz-Timme, S. Aspartic acid racemisation in purified elastin from arteries as basis for age estimation. Int. J. Legal Med. 2010, 124, 269-275.

(12) Ritz-Timme, S.; Laumeier, I.; Collins, M. Age estimation based on aspartic acid racemization in elastin from the yellow ligaments. Int. J. Legal Med. 2003, 117, 96-101.

(13) Cloos, P. A.; Fledelius, C. Collagen fragments in urine derived from bone resorption are highly racemized and isomerized: a biological clock of protein aging with clinical potential. Biochem. J. 2000, 345, 473-480.

(14) Ohtani, S.; Matsushima, Y.; Kobayashi, Y.; Yamamoto, T. Age estimation by measuring the racemization of aspartic acid from total amino acid content of several types of bone and rib cartilage: a preliminary account. J. Forensic Sci. 2002, 47, 32-36.

(15) Sivan, S. S.; Van, El. B.; Merkher, Y.; Schmelzer, C. E.; Zuurmond, A. M.; Heinz, A.; Wachtel, E.; Varga, P. P.; Lazary, A.; Brayda-Bruno, M.; Maroudas, A. Longevity of elastin in human intervertebral disc as probed by the racemization of aspartic acid. Biochim. Biophys. Acta, Gen. Subj. 2012, 1820, 1671-1677.

(16) Fisher, G. H.; D’Aniello, A.; Vetere, A.; Cusano, G. P.; Chavez, M.; Petrucelli, L. Quantification of D-aspartate in normal and Alzheimer brains. Neurosci. Lett. 1992, 143, 215-218.

(17) Kaneko, I.; Morimoto, K.; Kubo, T. Drastic neuronal loss in vivo by beta-amyloid racemized at $\operatorname{Ser}(26)$ residue: conversion of non-toxic [D-Ser(26)] beta-amyloid $1-40$ to toxic and proteinaseresistant fragments. Neuroscience 2001, 104, 1003-1011.

(18) Young, G. W.; Hoofring, S. A.; Mamula, M. J.; Doyle, H. A.; Bunick, G. J.; Hu, Y.; Aswad, D. W. Protein L-isoaspartyl methyltransferase catalyzes in vivo racemization of Aspartate- 25 in mammalian histone H2B. J. Biol. Chem. 2005, 280, 26094-26098.

(19) Roher, A. E.; Lowenson, J. D.; Clarke, S.; Wolkow, C.; Wang, R.; Cotter, R. J.; Reardon, I. M.; Zurcher-Neely, H. A.; Heinrikson, R. L.; Ball, M. J.; Greenberg, B. D. Structural alterations in the peptide backbone of beta-amyloid core protein may account for its deposition and stability in Alzheimer's disease. J. Biol. Chem. 1993, 268, 30723083.

(20) Fujii, N.; Tajima, S.; Tanaka, N.; Fujimoto, N.; Takata, T.; Shimo-Oka, T. The presence of D-beta-aspartic acid-containing peptides in elastic fibers of sun-damaged skin: a potent marker for ultraviolet-induced skin aging. Biochem. Biophys. Res. Commun. 2002, 294, 1047-1051.

(21) Ritz-Timme, S.; Laumeier, I.; Collins, M. J. Aspartic acid racemization: evidence for marked longevity of elastin in human skin. Br. J. Dermatol. 2003, 149, 951-959.

(22) Mori, Y.; Aki, K.; Kuge, K.; Tajima, S.; Yamanaka, N.; Kaji, Y.; Yamamoto, N.; Nagai, R.; Yoshii, H.; Fujii, N.; Watanabe, M. UV Birradiation enhances the racemization and isomerizaiton of aspartyl residues and production of Nepsilon-carboxymethyl lysine (CML) in keratin of skin. J. Chromatogr. B: Anal. Technol. Biomed. Life Sci. 2011, 879, 3303-3309.

(23) Hashimoto, A.; Nishikawa, T.; Oka, T.; Takahashi, K.; Hayashi, T. Determination of free amino acid enantiomers in rat brain and serum by high-performance liquid chromatography after derivatization with N-tert.-butyloxycarbonyl-L-cysteine and o-phthaldialdehyde. J. Chromatogr. B: Biomed. Sci. Appl. 1992, 582, 41-48.

(24) Hooi, M. Y.; Truscott, R. J. Racemisation and human cataract. D-Ser, D-Asp/Asn and D-Thr are higher in the lifelong proteins of cataract lenses than in age-matched normal lenses. Age 2011, 33, $131-141$. 
(25) Zhang, J.; Yip, H.; Katta, V. Identification of isomerization and racemization of aspartate in the Asp-Asp motifs of a therapeutic protein. Anal. Biochem. 2011, 410, 234-243.

(26) Adams, C. M.; Zubarev, R. A. Distinguishing and quantifying peptides and proteins containing $\mathrm{D}$-amino acids by tandem mass spectrometry. Anal. Chem. 2005, 77, 4571-4580.

(27) Tao, Y.; Quebbemann, N. R.; Julian, R. R. Discriminating Damino acid-containing peptide epimers by radical-directed dissociation mass spectrometry. Anal. Chem. 2012, 84, 6814-6820.

(28) Tao, Y.; Julian, R. R. Identification of amino acid epimerization and isomerization in crystallin proteins by tandem LC-MS. Anal. Chem. 2014, 86, 9733-9741.

(29) Jia, C.; Lietz, C. B.; Yu, Q.; Li, L. Site-Specific Characterization of d-Amino Acid Containing Peptide Epimers by Ion Mobility Spectrometry. Anal. Chem. 2014, 86, 2972-2981.

(30) Pang, X.; Jia, C.; Chen, Z.; Li, L. Structural Characterization of Monomers and Oligomers of D-Amino Acid-Containing Peptides Using T-Wave Ion Mobility Mass Spectrometry. J. Am. Soc. Mass Spectrom. 2017, 28, 110-118.

(31) Jeanne Dit Fouque, K.; Garabedian, A.; Porter, J.; Baird, M.; Pang, X.; Williams, T. D.; Li, L.; Shvartsburg, A.; Fernandez-Lima, F. Fast and Effective Ion Mobility-Mass Spectrometry Separation of dAmino-Acid-Containing Peptides. Anal. Chem. 2017, 89, 1178711794.

(32) Jansson, E. T. Strategies for analysis of isomeric peptides. J Sep Sci 2018, 41, 385-397.

(33) Fujii, N.; Sakaue, H.; Sasaki, H.; Fujii, N. A rapid, comprehensive liquid chromatography-mass spectrometry (LC-MS)based survey of the Asp isomers in crystallins from human cataract lenses. J. Biol. Chem. 2012, 287, 39992-40002.

(34) Maeda, H.; Takata, T.; Fujii, N.; Sakaue, H.; Nirasawa, S.; Takahashi, S.; Sasaki, H.; et al. Rapid survey of four Asp isomers in disease-related proteins by LC-MS combined with commercial enzymes. Anal. Chem. 2015, 87, 561-568.

(35) Lyon, Y. A.; Sabbah, G. M.; Julian, R. R. Differences in alphaCrystallin isomerization reveal the activity of protein isoaspartyl methyltransferase (PIMT) in the nucleus and cortex of human lenses. Exp. Eye Res. 2018, 171, 131-141.

(36) Friedrich, M. G.; Hancock, S. E.; Raftery, M. J.; Truscott, R. J. Isoaspartic acid is present at specific sites in myelin basic protein from multiple sclerosis patients: could this represent a trigger for disease onset? Acta Neuropathol. Commun. 2016, 4, 83. 